\title{
Digitally Enhanced Community Rescue Archaeology
}

\author{
Tom Dawson \\ School of History/SCAPE \\ University of St Andrews \\ tcd@st-andrews.ac.uk
}

\author{
Anna Vermehren \\ Timespan Museum \\ Helmsdale, Sutherland \\ director@timespan.org
}

\author{
Alan Miller, Iain Oliver \& Sarah Kennedy \\ School of Computer Science \\ University of St Andrews \\ (alan.miller,iao,sk383)@st-andrews.ac.uk
}

\begin{abstract}
Coastal erosion is causing the destruction of archaeological sites around the world. The problem is particularly grave in Scotland, where storms can cause many meters of land to be lost in a single event. Archaeological researchers from the University of St Andrews and the SCAPE Trust have worked with community groups to excavate sites before they are destroyed. Video was used to record the progress of the community rescue digs and interviews conducted with local group members. Additionally, photographs and artwork augmented the archaeological record, resulting in a wealth of information about the sites and the process of excavation.

The data has been used to make reconstructions of the sites as they were in the past. Visitors control avatars to explore the virtual worlds and to access videos, photographs, laser scans, 3D models and historic documents. This innovative approach to heritage interpretation allows the public to see the evidence behind reconstructions and to learn about the process of archaeological enquiry. For example, clicking on a plate of food reveals a summary of the environmental report which provided the evidence for diet. This allows an archaeological site report to be presented in a 3D environment, with various layers of information accessible to the explorer.
\end{abstract}

The group have set up their first installation in a dedicated room at Timespan Museum, Helmsdale. The evidence for the reconstruction comes from the sixteenth century Brora salt pan, excavated between 2007 and 2011 and destroyed in a storm in 2012 as well as the neighbouring township of Caen. Users manoeuvre the avatar either through a game controller or by body gestures recognised by a motion sensor. Multiple screens have been used to present a wraparound and immersive experience. The systems are built using OpenSource software and commodity hardware. They are designed to enable content to be augmented by non-technical specialists and allows cultural organisations and their participating audiences to create professional quality immersive environments at relatively low cost, and to develop their own interpretations of history and link them to wider narratives. In this way local communities are empowered to engage in the construction and transmission of their cultural heritage.

\section{INTRODUCTION}

Coastal erosion is recognised as a major and growing problem which threatens archaeological sites around the world. The challenge of unlocking the secrets that vulnerable sites hold can be met in part through mobilising local communities. The Scottish Coastal Archaeology and the Problem of Erosion Trust (SCAPE) has successfully worked with local groups on surveys and excavations at sites that will otherwise be destroyed. As well as retrieving conventional archaeological information used to investigate past societies, the projects have

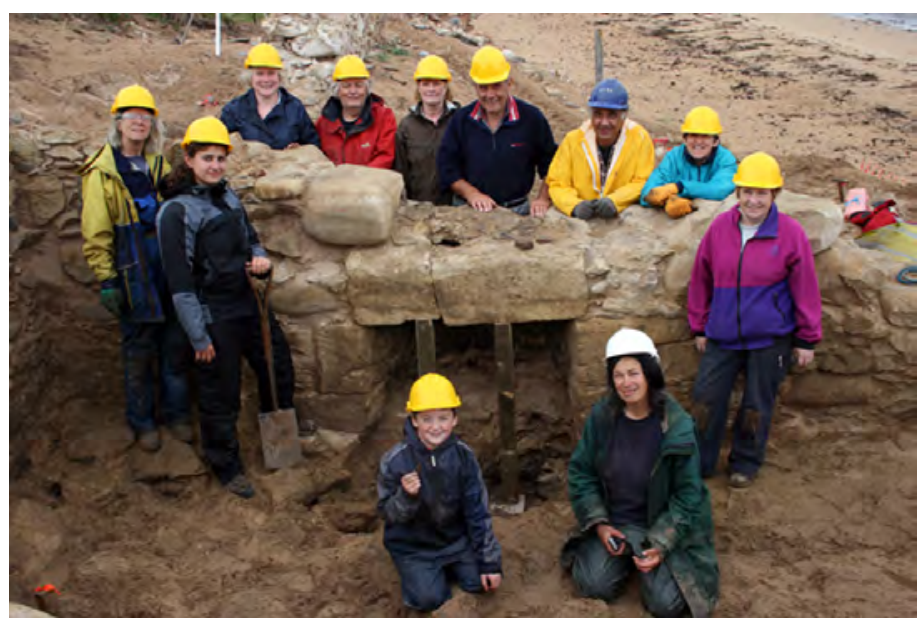

Fig. 1. Brora Salt Pan Community Excavation and fireplace.

recorded the process of investigation, capturing the memories, beliefs and ideas of modern societies and learning about how people today interact with the past.

Interest generated by engagement in the investigative process has been harnessed in the construction and propagation of local historical narratives (fig. 1). The spread of digital literacies and advances in commodity computational and communication devices offer opportunities to local communities at each stage of the process. This paper draws upon our concrete experiences in digitally enhancing specific community rescue archaeology projects and in presenting the results to the local community. Lessons are drawn about how emergent mobile and 3D technologies were used to bring together communities and professionals to successfully investigate and digitally preserve sites and create holistic reconstructions.

The core software together with the resources developed specifically for this project are open source and freely available. They run on commodity hardware meaning that costs are kept within the reach of local heritage centres. This research focusses on coastal erosion, an area of growing worldwide significance due in part to the increasingly unstable weather patterns associated with global warming. The discussion of engagement with local communities is relevant to digital reconstruction work more generally. The methodology outlined here is being applied in the Virtual Histories project developing reconstructions of coastal sites, in partnership with communities across Scotland.

Collaborations between SCAPE, Timespan Museum on the 


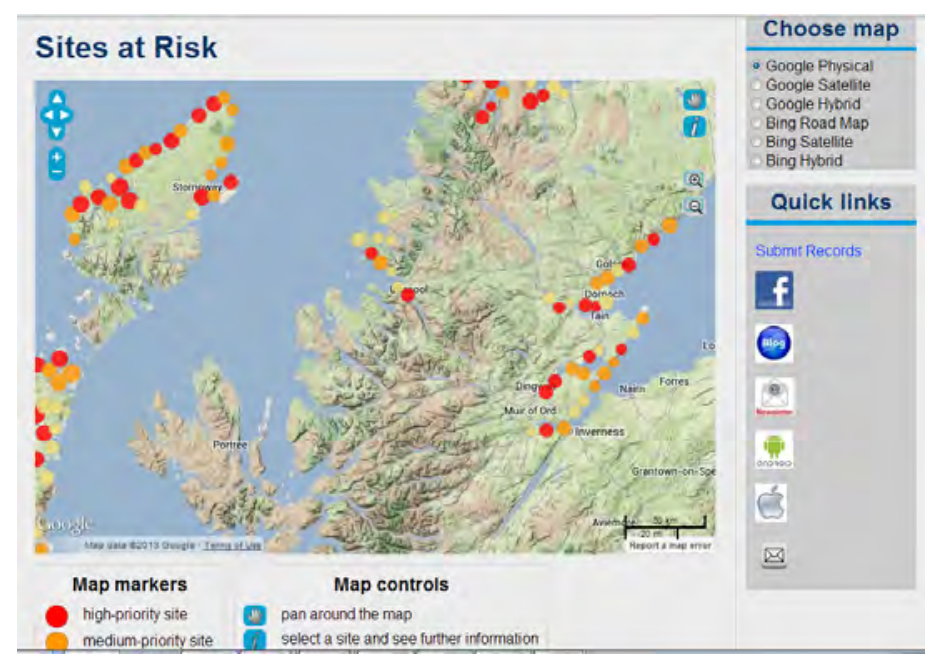

Fig. 2. Sites at Risk Interactive Web Site.

north east coast of Scotland and archaeologists and computer scientists at the University of St Andrews have enabled us to develop our approach. SCAPE and Timespan bring strong community participation to the reconstruction of three immersive digital resources local to the museum: a model of the Brora salt pans as found during excavation; a reconstruction of the buildings as they were in the 17th Century; and a reconstruction of the neighbouring Caen township. They are the subject for an installation at Timespan, accessible globally via the Internet; and through a mobile exhibition that tours schools and community groups.

The work in this paper makes contributions in three areas: recounts a successful methodology for mobilising local communities in rescue archaeology; makes the archaeological evidence available in innovative ways that enhance engagement; enables digital literacies to be applied to the construction and exploration of cultural heritage.

\section{GoAls And Challenges}

Scottish coastal archaeological sites present many challenges, which need to be met within the context of constrained resources. These include: documenting the location and condition of the resource, identifying and investigating high priority sites that are under threat and recording findings accurately whilst making data accessible to non-specialists. These challenges exist along the second longest coastline in Europe, much of which is sparsely populated and far from urban centres.

Surveys have shown that thousands of archaeological sites distributed along Scotland's $15,000 \mathrm{~km}$ of coastline are at risk from coastal erosion [1]. Although erosion is a natural process, changing weather patterns appear to be aggravating the problem. Trying to decide which of the numerous high priority sites to excavate is problematic, and the concept of 'public value" has been used to help make these decision [2]. Those sites that are valued locally, and which can be investigated in collaboration with community groups, are placed higher on the priority list than other threatened sites of similar archaeological value. Community participation in the project is a priority and local groups are involved in the planning stages of the recording project, as well as taking part in fieldwork. Open meetings are held in partnership with community organisations to develop understanding of the significance of the site, and workshops help create a strategy for site investigation. This in turn fosters local ownership of the project. Although collaborative projects between local volunteers and professional archaeologists bring their own challenges, there is much to be learned from local community members; and communication and dialogue between technical experts and volunteers can reveal a wealth of knowledge. For this reason, in addition to documenting the archaeological evidence, the excavation process itself is recorded, through print, photography, audio recording, artwork and other creative methods.

For threatened sites, the physical preservation of the remains is often impossible without the construction of costly coastal defences; survey and rescue excavation are frequently the preferred options. In order to aid recording, digital preservation through laser scanning or other techniques are employed. However, collecting data is not an end in its own right, there is also the challenge of enabling non-specialists to gain access to the digital models. Conventionally, fly-throughs have often been employed, but a more accessible method is to put the user in control, allowing them to explore the model and discover links to other resources themselves. Meeting this challenge transforms digital models from a valuable archive into an accessible part of a community's cultural heritage.

Possibly the biggest challenge (and, if met, the most valuable accomplishment) is for the rescue excavation to contribute organically to a deeper understanding of the local heritage, and as a result, to raise local pride and community cohesion. To achieve this, a dialogue between all participating professionals and volunteers should be undertaken to continuously construct a shared understanding of the past. This can lead to deep reflection and reassessment of local histories. Excavation reports require professional training to write and are often too technical for average public consumption. For community excavations, there is a need to make all information freely and easily accessible. The final challenge is to meet these requirements within tight cost constraints. Archaeological excavation and recording can be costly and the post excavation work is also expensive. Archaeological work at eroding sites is often financed using public money, and budgets are tight in the present economic climate. A means of reducing the costs of dissemination is therefore desirable.

\section{Preservation, Games and Virtual Worlds}

Three dimensional environments have been used to impressive effect to present cultural heritage for digital preservation, both through block buster computer games and in virtual world amateur scenarios. This section surveys the existing work in three fields and considers its relevance to community-led rescue archaeology.

The transmission of cultural heritage between generations contributes to the construction of shared understandings of the world. Both formal and informal education play their part in this process. Visits to historic monuments and museums contribute, as do multimedia presentations. Historical films like Braveheart provide powerful images which shape popular conceptions of the past. Computer games containing historic 

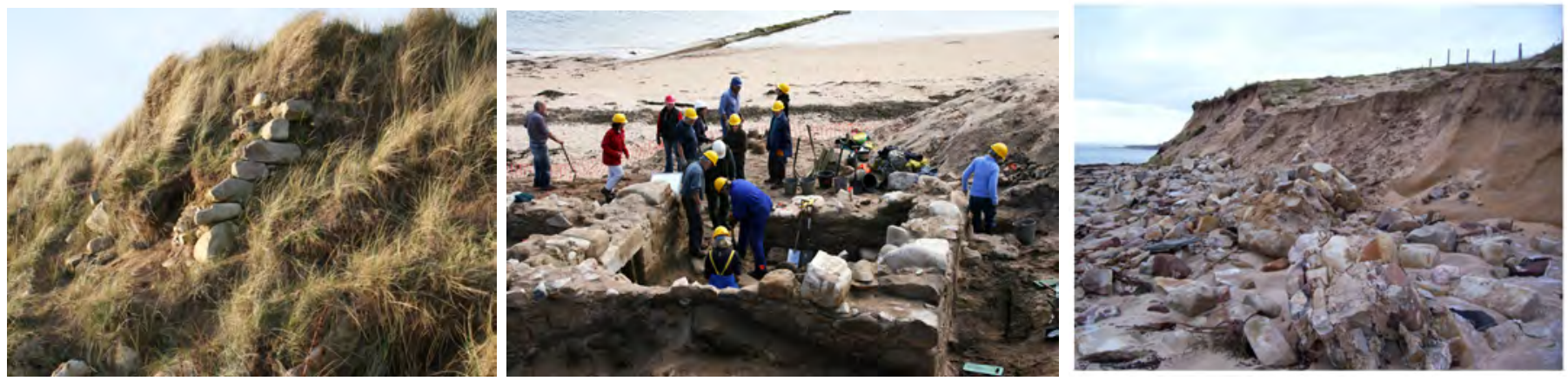

Fig. 3. Coastal erosion leads to site discovery.

Fig. 4. Community rescue excavation and recording.

Fig. 5. Site Destruction through coastal erosion.

content such as Civilisation [3], [4], Total War [5] and Assassins Creed, provide interactive environments which also shape the perceptions of their players. However, the entertainment industry is not constrained by requirements of authenticity as rigorously as the informal and formal education sectors.

In the past decade there has been tremendous growth in the use of 3D technologies for the digital recording of historic monuments. The Scottish Ten, a collaborative project sponsored by the Scottish Government and managed by Historic Scotland, has used laser scanning technology to create digital models of world heritage sites in Scotland and across the globe. Elsewhere Michelangelo's statue of David has been digitised along with other significant sites by Stanford University, and CyArk is building a digital resource of cultural heritage sites from across the globe. The digitisation of heritage sites has created a record that survives whatever degradation or disaster may befall the physical site, whether man made or natural. However, the amount of data gathered has constrained accessibility except through the presentation of passive fly-throughs.

A third area of relevant digital activity has been within virtual worlds, the most important being Second Life (SL). A virtual world is a $3 \mathrm{D}$ environment within which the user is represented by an avatar. The proxy of the avatar enables users to interact both with the environment and with other users, connected concurrently with a client. Second Life relies upon the public to generate content, consequently there are easy to use, yet high quality tools for building in-world. It is also possible to write scripts to develop interactive activities. Reconstructions within SL can be categorised into three types [6], environments that recreate the look and feel of a time period without reconstructing an authentic building; reconstructions of existing locations, such as Michelangelo's Sistine chapel and historical reconstructions such as Virtual Harlem.

The service model employed by SL constrains its functionality. Virtual land has to be rented, programming is limited to small scripts and the size and scale of buildings are limited. Reliance on Internet connectivity means that the user experience maybe degraded [7] or installations and portable exhibits are not possible. If funding for the rented land runs out the reconstruction is lost. In recent years an OpenSource alternative to SL, the OpenSimulator (OpenSim) [8] project has been developed. Organisations are able to host models on their own servers giving far more flexibility in deployment and the ability to host large land masses at minimal cost. Furthermore the systems are extensible, allowing powerful programming paradigms [9] to be used to develop custom systems and to provide a holistic historical experience.

The emergence of freely available 3D software, which supports user centred exploration, interactivity, multimedia, concurrent users and web connectivity offers not only the ability to create rich historic reconstructions but also the potential to engage communities in the process of $3 \mathrm{D}$ reconstruction, in the development of narratives linked to the reconstruction and to use existing digital literacies to explore them.

\section{Community Rescue Archaeology}

SCAPE and the University of St Andrews have been at the forefront of community rescue archaeology. Their latest venture, the Scottish Coastal Heritage @ Risk Project (SCHARP) is concerned with mobilising volunteers from local communities to track the state of known coastal archaeology at risk [10]. Information about sites at risk is held in a database and made accessible through a Sites at Risk Map (http://scharp.co.uk/sites-at-risk/) with clickable hotspots that reveal information about each site: (fig. 2). Thus volunteers can easily identify the location of sites and can edit and update information to report the present condition of vulnerable assets located within the highly dynamic coastal zone. The project has developed a mobile phone app which enables the public to access information and to provide updates via a simple multiple-choice form and by sending in photographs. The app utilises GPS to automatically and accurately identify location, and forms guide the user through the submission of photographs and descriptive text. This information is validated and updates made available on the Sites at Risk map. The second stage of the project asks communities to nominate sites valued locally and follow-up projects will be started at 12 of these during the three-year life of SCHARP.

SCHARP is a development of another SCAPE project Shorewatch [11]. This also set up community excavations at eroding sites, and one completed project was at the Brora Salt Pans. Figures 3, 4 and 5, illustrate the discovery, excavation and later destruction of the Brora salt pans site. We have used the results of this community excavation, to develop aspects of our rescue methodology. Brora's place in history as the industrial capital of the highlands is due to a small pocket of Jurassic rocks containing coal on the east coast of Sutherland. Coal was mined for hundreds of years, leading to the development of other industries, including three attempts at 


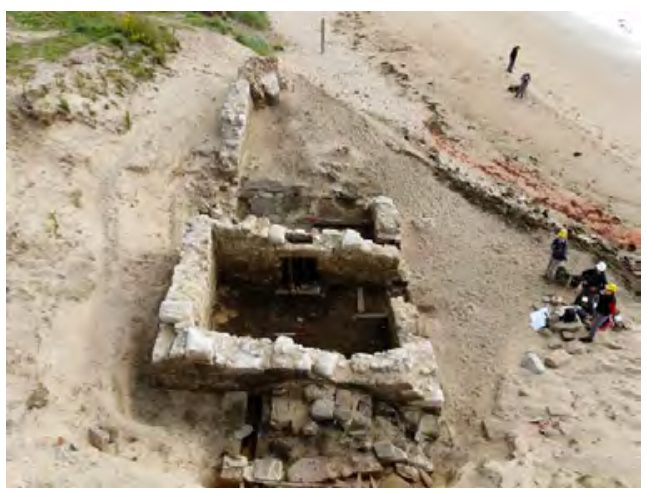

Fig. 6. Community excavation in progress.

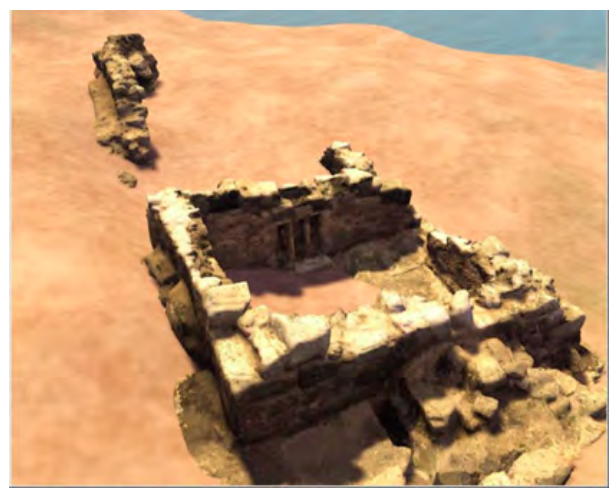

Fig. 7. Model of Excavation in Virtual World.

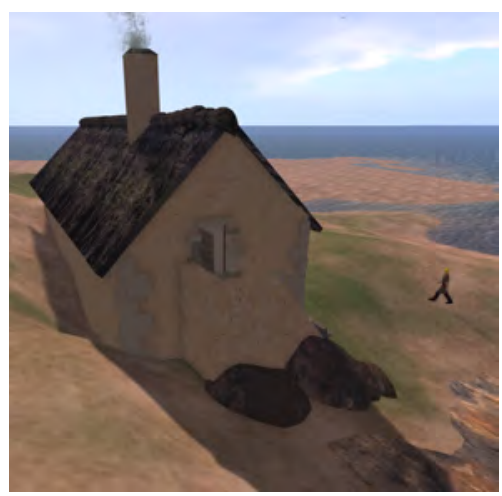

Fig. 8. Reconstruction of excavated building. salt production between 1598 and the mid-nineteenth century. In 2004, members of the Clyne Heritage Society and the North of Scotland Archaeology Society recorded a long wall of an eroded building on the beach as part of the Shorewatch project [12].

The following year, a more comprehensive assessment of the archaeological features visible was carried out. The results of this initial work clearly showed the perilous state of the remains due to rapid coastal erosion. As a result, a project supported by Historic Scotland and managed by SCAPE saw a team of archaeologists working with local volunteers, Excavations were undertaken at the site of the New Salt House [13], [14] and the Old Salt House [14], located less than $100 \mathrm{~m}$ apart, but separated in time by 150 years.

The Old Salt House had been buried within the dune and only one corner of the building was visible before the excavation commenced. This had started to erode rapidly and heavy machines were used to uncover the site. The building was remarkably well preserved, with walls standing over two metres high and two separate rooms uncovered. The building included an intact fireplace within the partition wall, with graffiti type engravings on the lintel and sides, and food debris indicating a strong presence of fish in the workers diet.

In the winter of 2012/2013 much of the site was destroyed by the sea, and it is expected that the rest of the site will be destroyed in the near future. This means that the data gathered through the excavation has an added importance. The record includes artefacts and ecofacts, point cloud data of the excavated building retrieved using a Leica laser scanner, measured drawings, photographs, video recording of the excavation and interviews with the participants and other members of the local community.

\section{Digital Preservation AND ReCONSTRUCTION}

In 2012, work started on creating a digital 3D immersive environment for Timespan Museum to commemorate life in the region. This includes a reconstruction of the sixteenth century salt pans as they would have been when they were functioning, derived from the laser scan data.

The exhibition enables existing digital literacies to be used to explore data related to the excavation in visual and intuitive ways. A user is represented by an avatar that may be controlled by an XBox controller, natural movements or a key board and mouse. The models may be accessed over the Internet, within Timespan exhibition space or as part of a touring LAN Exhibition. This enables the wider local community to engage with the interpretation of the past made possible through the excavation work.

The first digital model of the site as it was during excavation was created from the laser-scan and topographic data of the surrounding landscape. The excavation site is located upon a $0.5 \mathrm{~km}^{2}$ island and the model has four main components: the landscape, a mesh model of the excavation, interactive web pages and embedded video. The topographic data gave one point per ten meters which was interpolated to give one point per meter and scaled onto an 8 bit bitmap to minimise quantization error. The bitmap was scaled within virtual world space. Textures were used to establish appropriate base colours by height, the main distinctions being between the sea, the sandy beaches and grasslands. The points cloud data was exported into XYZ format and imported into Meshlab. Here it was separated into 12 segments to enable ease of processing and to facilitate uploading into the Virtual World. The meshes were then scaled, placed within the landscape and reconnected as shown in fig. 7 .

Information about the process of the excavation is presented within a series of web pages that are associated with the site model illustrated in figs. 9, 10 and 11. These pages are either embedded as textures on surfaces in the model or embedded in scripts which load into a web browser. The user may choose whether the web browser opens within the virtual world client or as a separate window. Video of community participants have been made available for viewing on surfaces within the digital site. By default, when an avatar points at the surface containing a link to the video, an on "Click event" occurs, causing a full screen canvas to be created. Playback of the video can then be controlled using the mouse. If the image is minimised, the video will continue to play within the surface it is embedded in. The range of audio associated with a video can be adjusted and the avatar can move around and change the field of view.

Having explored the excavation process, the visitor teleports across time to re-emerge at the same location at the end of the 16th Century. The reconstruction of Brora Salt Pan offers a different perspective on the site (fig. 8). The visitor 

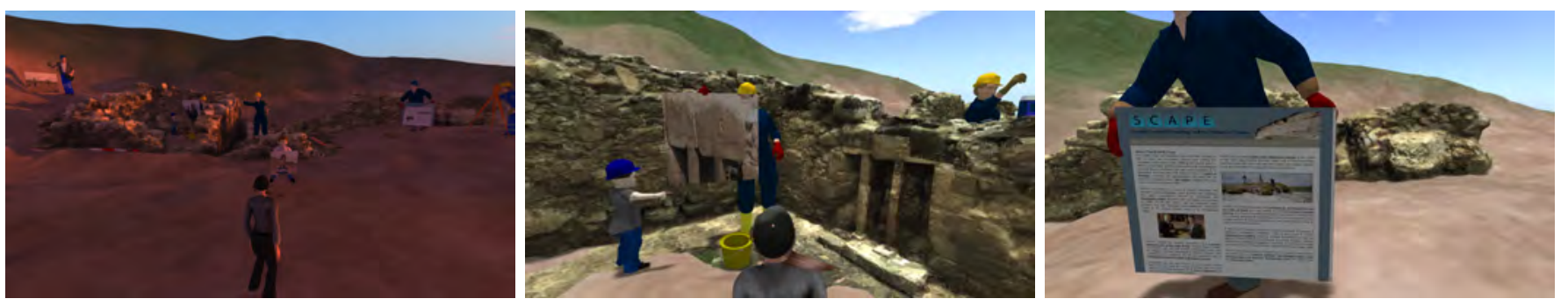

Fig. 9. Avatar exploring onsite interactive content. Fig. 10. Fireplace with commentary by Jacquie. Fig. 11. Limekiln and SCAPE website.

is now standing at the same spot, but 400 years earlier as illustrated in figs. 12, 13 and 14. The reconstruction is based upon evidence uncovered during the excavation and the wider historical record. The buildings are intact and the coastline is further away, yet to be affected by erosion. The topographic data has been remodelled to reflect the old coastline. The buildings themselves are intact.

The embedding of media in the historical model and links to web content enable visitors to examine evidence for the reconstruction and to explore wider explanatory materials and narratives. For example the reconstruction of the fireplace includes links to explanations of the graffiti inscribed upon it; while videos demonstrate the process of salt creation in the pans. Historical documents detail attempts by the Sutherland Estate to establish a panning industry.

The reconstruction model also includes details derived from the post-excavation analysis of the recovered material. For example, window glass was recovered, and as it is very unusual for buildings to have glass at this period in Highland Scotland, analysis was needed to prove that the glass was contemporary. This was done by using X-Ray Fluorescence techniques to examine the chemical properties of the material and detailed scientific analysis showed the glass was contemporary. In order to help the public understand this process, tabbed web pages have been embedded within the model. Clicking a window allows access to several pages of information. One explains the problem, another shows examples of seventeenth century windows; and other pages explain the scientific procedure and present the detailed scientific report. The tabs are clearly labelled and progress from simple to technical. The user chooses the level of detail they engage with; most people may not wish to see the full evidence, but they may want to understand the process of analysis. Presenting the evidence in this way means that detailed archaeological reports can be displayed in new and exciting ways, and the evidence that has led to the creation of the reconstruction is available for all to view.

\section{SyStem SUPPORT FOR EXPLORING THE PAST}

This project utilises emergent 3D technologies to bring communities together with historians, public engagement professionals and technical specialists in the co-creation and curation of reconstructions of the past, which are based primarily upon the output of a community archaeological excavation. These digital assets are accessible using established digital literacies. They provide gateways to evidence, commentary and interpretation which in turn are based upon archaeological and historical evidence. The digital assets also link together communities enabling each to discover and reflect on common themes. In recent years an open-source virtual world technology has emerged enabling organisations to host their own environments giving flexibility in deployment and cost effectiveness. Furthermore these systems are extensible, allowing powerful programming paradigms to be used to develop custom systems and provide an holistic historical experience. Hyper-grid technology allows independently hosted servers to be connected together enabling avatars to teleport between models hosted in different physical locations. Links from virtual world content to the web and social media allows the characters and objects within 3D models to act as an interface to and context for web pages and social media.

The OpenSimulator (OpenSim) project has developed a 3D application server which integrates well with web based applications, is compatible with Sketch Up and 123D Catch and has the potential to enable many of the challenges facing the digital empowerment of community rescue archaeology to be met.

At the heart of an OpenSim web application is a 3D environment, which users explore through the proxy of an avatar. It is easy and intuitive to explore and supports multiple concurrent access. The system is freely available and may be configured in different ways to suit the full range of likely cases. Content may be developed in-world using easy to use development tools, and it accepts input in the COLLADA mesh format making it compatible with the full range of 3D development environments from Sketchup and 123D Catch to Blender, AutoCAD and Maya. The environment is easily accessible from the WWW and WWW resources are accessible from within the virtual world. OpenSim contains tools for inworld development and has facilities for uploading models, images, sounds and landscape data. The modelling takes place while the system is running. This facilitates collaboration and the bringing together of archaeologists, heritage centre professionals, community volunteers and system developers. The technology supports collaboration and empowers digital rescue archaeology. The system is open source and designed to be extensible. Servers may be hosted in locally and configured to meet specific needs.

The virtual world system is made up of three discrete components: the client, the simulations and services:

A client allows the user to connect to a server and provides an interface for viewing and interacting with content. It provides control over how the data is displayed, for example lighting, view distance and fidelity of the data are all configurable. The client provides an interface for uploading content into the virtual world. Just as there are multiple Web browsers (Internet 


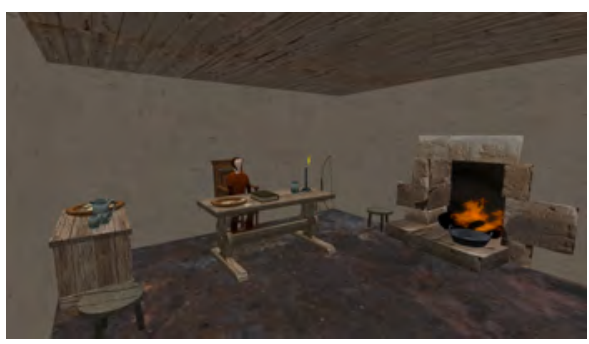

Fig. 12. Fireplace in reconstruction.

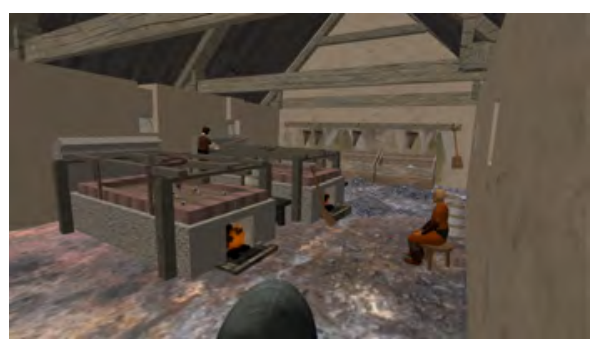

Fig. 13. 16th Century Salt Pan.

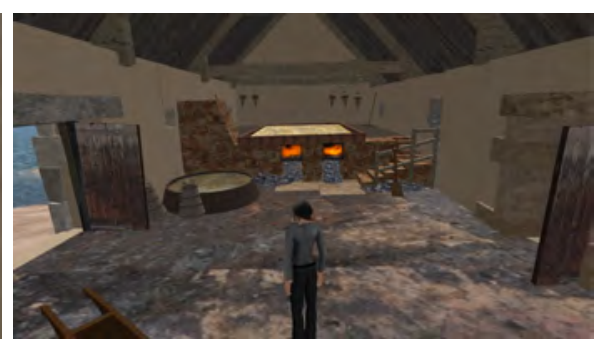

Fig. 14. 18th Century Salt Pan.
Explorer, Firefox, Safari and Chrome), there are several clients that may be used to access server these include: the SL client, Firestorm, Imprudence and Hippo.

Services provide the information required by a simulation to both run the simulation and to control access. These may be located on the same machine as the simulation, on a single machine or distributed across multiple servers. Services include user authentication, and provide content to populate the regions with.

Each simulation is responsible for keeping track of all the objects and avatars contained within a virtual space. It receives updates from and distributes updates to clients and makes use of services, to retrieve information about avatars buildings and landscapes. Simulations are made up of discrete functional components which enable control over the weather, the physical structure of the space, the actions of avatars, sound and interactions with the WWW. Thus both tangible and intangible culture can be represented in-world. Regions represent a discrete area of land and adjacent regions may be located on separate servers. A user is able to view the contents of and walk between contiguous regions. Consequently, allowing large land spaces may be constructed. The Environment is controlled by adjusting wind, clouds, water, visibility, the sun, moon and the time of day. A space maybe experienced at midday, during a summer sunrise, a misty afternoon or a moonlit night. Server side defaults may be set to define an intended experience. The landscape heights may be set by uploading a grey scale image map where shade tone defines height, it may then be transformed using server side commands and adjusted using client side tools.

The extensibility of OpenSIM means that it can support multiple modes of control. The standard mode of interaction is with a keyboard and mouse. This is highly configurable, with the possibility of assigning commands to keys and changing the behaviour of mouse movements. A popular configuration is to control movement with arrow keys and orientation with the mouse. This mode of operation offers accurate control appropriate for development and facilitates communication with others using the keyboard for chat.

A game controller offers easy control of movement and direction of view. For example of an X-Box controller has three 2D joysticks, which map to Move (forward, backward, turn left, turn right), Mouse-look and mouse. There are also large triggers, which Map to y up and down. Shoulder buttons are mapped to right and left click. Zooming the camera in and out is mapped to two function buttons.

We have also extended OpenSim to receive input from a
Microsoft Kinect. This allows natural gestures to be used to select and active features in the interface and to control avatar movement.

\section{DePloyment, Use and EVALUation}

For the reconstructions to be of value it is necessary for the systems to deliver a high quality of user experience. Systematic study of systems aspects of OVWs underpins the work described in this paper. This has shown that OpenSim is able to deliver an appropriate quality of user experience using commodity hardware based systems.

In [15] users quality of experience is analysed. Start up delay, frame rate and system responsiveness are each identified as key performance metrics, whilst resolution, quality of rendered environment, and quality of lighting are key quality metrics. There is an inverse relationship between performance and quality metrics: for example switching off shadows will result in a higher frame rate. Observation of systems in use in multiple environments combined with system measurements lead to the simple heuristic that it is best to sacrifice performance until frame rates reach around 15 frames per second after which quality can be increased. A typical virtual world system is made up of a clients, network and one or more servers. It is important that balanced systems are used and the relationship between Client, Server and Network limitation are understood. The time it takes to download resources at the start of a session is shown in fig. 15; the frames per second generated by a server for increasing numbers of avatars in fig. 16 and the distribution of client side frames per seconds for the range of client resolutions in fig. 17.

A network measurement study reported in Oliver et al [16] demonstrates that virtual world client benefits from up to 1 megabit per second and can operate effectively with around 100 to $150 \mathrm{kbit} / \mathrm{sec}$ for each user. In Mongoose [17] a traffic management system for OpenSim is presented which adapts automatically to network condition and prevents high priority avatar control traffic from interfering traffic. This improves user Quality of Experience in low bandwidth scenarios. Further studies demonstrate that a modern i7 based server can safely handle up to 25 regions and that a single region can receive around 50 avatars before being overloaded [15]. These measurement-based lab experiments are backed up through measurement and observation of SL and OpenSim being used in the wild [16], [18], [19].

In summary an OpenSim based system is able to handle many simultaneous users and operate in environments with as little as a megabit per second. This makes it appropriate for 

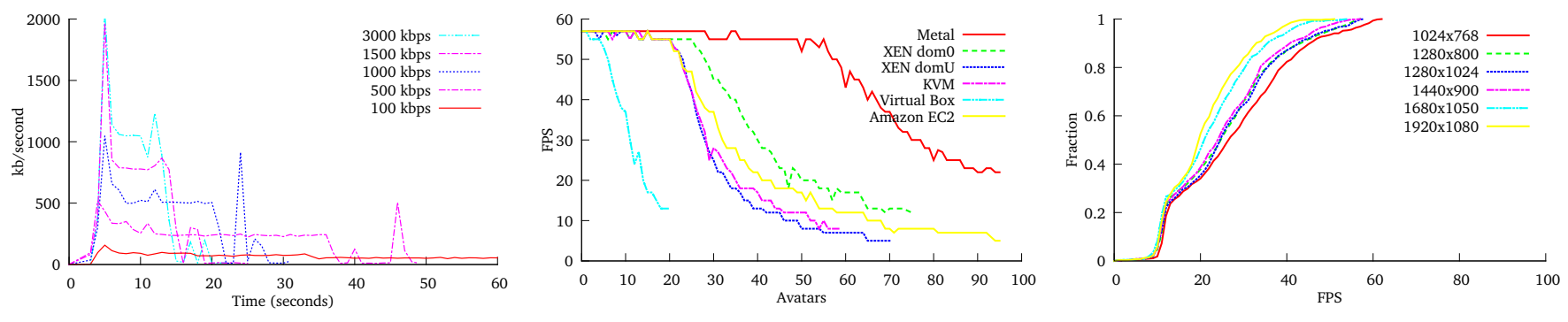

Fig. 15. Start up time and network bandwidth.

Fig. 16. Server frame rate and number of avatars. Fig. 17. Client frame rate and screen resolution.

small to medium size heritage centres and portable exhibitions involving up to 100 simultaneous participants. It is also feasible to connect on a good mobile broadband connection. Thus the system is economical and fit for purpose for the use cases discussed in this paper.

A Live Internet service consists of: the full historic scenes, including models, historic characters and sound tracks along with links to supporting evidence and community commentary, embedded in interconnected virtual world regions; the digital models embedded in web pages; the archaeological evidence and community generated multi-media commentaries are also accessible directly from web pages, connections with social media will enable virtual visitors to add commentary. Users may create accounts for the Virtual World service via a simple web interface and full access is achieved through freely available client software (Firestorm). They may also be accessed via the Hyper-GRID an interconnected network of Virtual World GRIDS with over 20,000 regions. A single in world portal provides easy navigation to each of the scenes. In addition web page documentation will be created for each of the scenes and 3D models of each scene embedded in the web page. Servers are hosted in a bespoke server room, with appropriate cooling, maintenance and general care. This provides high speed access to the JANET network. It provides streamlined access to the digital models, historic scenes, archaeological evidence, multimedia community generated content and connected social media. It is available 24 hours a day seven days a week from computers connected to the Internet.

Each resource has been packaged into a self-contained stand-alone system, with virtual world client and server, local web server and supporting data. It is available for download as a single file repository. After download, when the system is unpacked it may be run, with a single click on a local system without the need for network access. The downloaded system may be edited, modified and re-factored and re-used as required.

The reconstruction is also hosted as part of an installation in the Timespan museum shown in fig. 18. This configuration provides a 6 megapixel display $(3 * 1980 x 1080) 300$ inch display, made up of three panels, the images of the three panels are precisely synchronised. This consists of 3 HD 1080p Projectors, Microsoft Kinect, Installation computer: a quad core haswell i7 processor, Nvidea GTX 780 graphics card and $16 \mathrm{~GB}$ of memory.

This has proved capable of delivering powerful experience as evidenced by:

One of my own ancestors was cleared from Caen. The archae- ological remains of this era lie undisturbed in our Strath but to see life as it was then is to stretch the imagination a bit too far. I can now sit in Timespan (or at home) and take my avatar through the township into the long-houses, corn-drying kiln and byres to see what my own ancestors saw. This is a surreal experience let me tell you! In Timespan, we have been looking for ways to attract new audiences to learn about our heritage, which can be considered by some as a bit dry and dusty and just for the older folk. Here we have up to date technology that children and young adults are familiar with, regularly use and work with, so bringing our heritage fully into the 21st century. Jean Sergeant Caen Descendent

A roving interactive exhibition has been established which enables the reconstructions to be taken out into the community. It enables groups of thirty to explore the historic scenes concurrently, teleporting between them. The local server means that Internet access is not required increasing deployment opportunities. The exhibition consists of a server, 6 portable workstations with X-Box controllers, Kinects, high resolution screens, projectors and supporting materials. It is of particular value in local schools facilitating integration of the reconstructions with local units of the curriculum of excellence. The exhibition has been set up in community centres, schools and other public buildings. School students exploring a reconstruction are shown in fig. 19 and a cloud of words drawn from their descriptions is shown in fig. 20. As Alex Duff, Education Manager, Emerging Technologies, Education Scotland comments:

The pupils took to the simulation like fish to water. They were up and running within minutes ... The energy and sense of investigation interaction with the simulation was very impressive.

Each of the three modes of deployment are appropriate in different circumstances and have different characteristics: the installation provides a powerful immersive experience, the mobile exhibit combined with X-Box controllers aligns well with existing digital literacy and the live Internet service provides the convenience of anytime anywhere access. This is in turn possible because of the extensibility of OpenSim.

\section{CONCLUSION}

As global warming leads to more turbulent weather patterns there is a growing threat to many cultural heritage sites located on the coast. In Scotland which has 15,000 km of coastline, this threat is particularly acute.

Developments in commodity technology that is readily available, including smart phones, laptops and computers, 

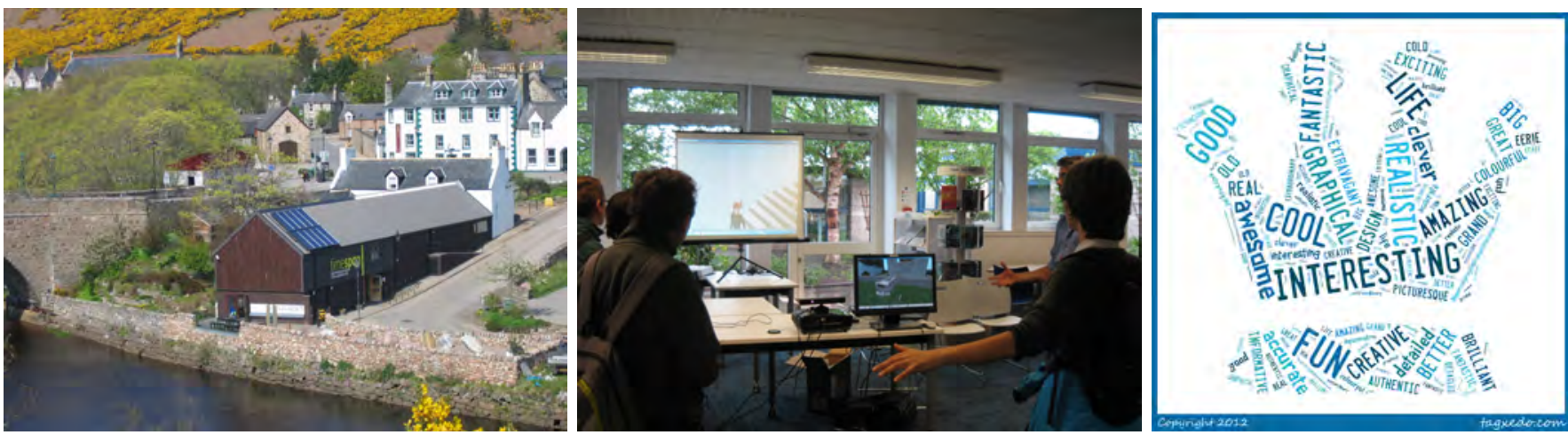

Fig. 18. Timespan Museum site of installation.

Fig. 19. School students controlling avatar with Kinect. Fig. 20. School student word crowd.

means that it is possible to construct powerful new mobile applications and 3D environments. This is coupled with a revolution in the digital literacies that are prevalent in the population at large. These developments make possible the advancement of community rescue archaeology and facilitate the development of applications which enable heritage managers, domain specialists, technologists and local communities to cooperate together. The use of open access 3D models publicises results and allows participants and the wider public to have greater access to knowledge about the scale of the problem and the methods for mitigation. A growing awareness and a sharing of results of excavation in new and exciting ways, will lead to greater efforts to save those parts of our heritage that will otherwise be destroyed.

This paper has discussed how technology, communities and professionals interact in the identification of sites, the investigation of the archaeology, the reconstruction of monuments and the communication of results. Taken together, this methodology enables the mobilisation of communities in the preservation of physical heritage and the construction of local narratives which give them ownership of their heritage. We have demonstrated how the themes of community rescue archaeology and digital reconstruction can work together to stimulate interest in archaeological investigation, preserve sites that are under threat from coastal erosion and enable the reevaluation of popular local historical understandings. The work reported in this paper demonstrates that the goals of coastal heritage preservation and stimulating interest in archaeology can be realised through harnessing local participation and emergent immersive technologies. Through doing so, the value of local cultural heritage is deepened. This has global significance at a time when the threat of coastal erosion to heritage is increasing due to unstable weather patterns associated with global warming.

\section{REFERENCES}

[1] P. J. Ashmore, "Archaeology and the coastal erosion zone," in Coastal Archaeology and Erosion in Scotland. Historic Scotland, Edinburgh, 2005.

[2] T. Dawson, "Erosion and coastal archaeology in scotland - managing a natinal problem, with examples from the Uists," Journal of the North Atlantic, 2013.

[3] B. Frye and A. M. Frager, "Civilization, colonization, simcity: Simulations for the social studies classroom," Learning and Leading with Technology, vol. 24, no. 2, pp. 21-23, 32, 1996.
[4] Firaxis Games and Westlake Interactive, "Sid Meier's Civilization III," Infogrames http://www.civ3.com/, 30 October 2001.

[5] The Creative Assembly, "Medieval: Total war," Activision, p. Computer Game, 20 August 2002.

[6] L. M. Sequeira and L. C. Morgado, "Virtual archaeology in second life and OpenSim," Journal of Virtual World Research, vol. VI, p. No1, 2013.

[7] M. Claypool and K. Claypool, "Latency and player actions in online games," Commun. ACM, vol. 49, no. 11, pp. 40-45, 2006.

[8] A. Frisby, MingChen, S. Dague, Tedd, justincc, Teravus, J. Berntsson, C. Krinke, D. Scofield, Dahlia, Mikem, M. Thielker, H. Horwitz, I. Brown, C. Lopes, Nlin, and A. R. S. Valadares, "Opensim source (version 0.6.7)," http://dist.opensimulator.org/opensim-0.6.7-release.tar.gz, 14 October 2009, [Online; accessed 10-November-2010].

[9] J. McCaffery, A. Miller, and C. Allison, "Extending the use of virtual worlds as an educational platform - network island: An advanced learning environment for teaching internet routing algorithms," in CSEDU (1), 2011, pp. 279-284.

[10] T. Dawson, "Erosion and coastal archaeology: Evaluating the threat and prioritising action," in HOMER Conference Proceedings, BAR International series, 2013.

[11] S. Fraser., S. Gilmour, and T. Dawson, "Monitoring scotland's coastal archaeology," in Coastal Archaeology and Erosion in Scotland. Historic Scotland, 2005.

[12] J. Aitken and J.Hooper, "Back beach, brora, highland (clyne parish), excavation," Discovery Excavation Scotland, vol. 8, 2007.

[13] J. Hooper, "Back beach brora excavation," Discovery Excavation Scotland, vol. 9, 2008

[14] J. Hambly, "Back beach brora, highalnd," Discovery Excavation Scotland, vol. 11, 2010.

[15] I. A. Oliver and A. H. D. Miller, "Towards the 3d web," in Proceedings of the 27th IEEE Conference on Advanced Information Networking and Applications, 2013.

[16] I. A. Oliver, A. H. Miller, and C. Allison, "Virtual worlds, real traffic: interaction and adaptation," in Proceedings of the first annual ACM SIGMM conference on Multimedia systems, 2010.

[17] I. Oliver, A. Miller, and C. Allison, "Mongoose: throughput redistributing virtual world," in Proceedings of the 21st IEEE International Conference on Computer Communication Networks (ICCCN 2012) IEEE, International Conference on Computer Communication Networks (ICCCN 2012), 2012.

[18] C. Allison and A. Miller, "Growing the use of virtual worlds in education: an OpenSim perspective," in Proceedings of the iED Europe, 2012.

[19] S. Kennedy, L. Dow, A. Miller, and R. Fawcett, "Living history with Open Virtual Worlds: Reconstructing St Andrews Cathedral as a stage for historic narrative," in Proceedings of iED Europe, 2012. 